\title{
CETP inhibition in the REVEAL trial: could we aim higher?
}

Amirhossein Sahebkar ${ }^{1,2,3}$, Dimitri P. Mikhailidis ${ }^{4}$, Maciej Banach ${ }^{5,6}$

${ }^{1}$ Halal Research Center of IRI, FDA, Tehran, Iran

${ }^{2}$ Biotechnology Research Center, Pharmaceutical Technology Institute, Mashhad University of Medical Sciences, Mashhad, Iran

${ }^{3}$ Neurogenic Inflammation Research Center, Mashhad University of Medical Sciences, Mashhad, Iran

${ }^{4}$ Department of Clinical Biochemistry, Royal Free Hospital Campus, University College London Medical School, University College London (UCL), London, United Kingdom ${ }^{5}$ Department of Hypertension, WAM University Hospital in Lodz, Medical University of Lodz, Lodz, Poland

${ }^{6}$ Polish Mother's Memorial Hospital Research Institute (PMMHRI), Lodz, Poland

Submitted: 1 June 2018

Accepted: 3 June 2018

Arch Med Sci 2020; 16 (5): 1229-1230

DOI: https://doi.org/10.5114/aoms.2020.97968

Copyright @ 2020 Termedia \& Banach

We have read the recent data on the Randomized EValuation of the Effects of Anacetrapib through Lipid-modification (REVEAL) trial with great interest [1]. Apart from the well-controlled baseline low-density lipoprotein cholesterol (LDL-C) levels, there are at least two potential factors that could have minimized the effect size of anacetrapib in the trial [1]. First, according to the population-based studies, both very low and very elevated high-density lipoprotein cholesterol (HDL-C) levels are associated with increased mortality [2, 3]. In the REVEAL study [1], a minor proportion of individuals had a low HDL-C at baseline. Since this category is the one that could benefit from any putative effect of HDL-C being raised by anacetrapib, it is possible that the risk of mortality would be lower if the drug were tested in a population with very low baseline HDL-C levels. Second, the risk reduction in coronary events with anacetrapib was expected based on the magnitude of non-HDL-C, but not LDL-C (beta-quantification assay) reduction. This might reflect the superiority of $L D L$ particle number over LDL-C for determining cardiovascular (CV) risk [4, 5], and could explain the small effect of anacetrapib, which may mainly affect the cholesterol content of LDL particles rather than their removal by LDL receptors.

The results of the REVEAL study also raise a critical question on their clinical relevance taking into account only $9 \%$ relative risk reduction (RRR) and 1\% absolute risk reduction of the primary endpoint [1]. There was also no significant effect on major atherosclerotic events [1]. Moreover, opposite to statin therapy [6], as well as combination therapy with evolocumab [7], for which the significant effect on CV outcomes appears after 12 months, in the case of anacetrapib significant reduction of main CV outcomes was observed after 3 years [1]. Therefore, currently the main question is which population might benefit the most from anacetrapib as an add-on to statin therapy. The good safety profile and very good adherence to therapy (almost 90\%) suggest that one of the groups might be non-adherent patients (even over 60\% after 2 years of statin therapy) [8] as well as those with statin intolerance [9, 10]. Finally, some other subgroups might benefit from anacetrapib, e.g. elderly patients at high and very high CV risk, as the mean age in the study was 67, with a high proportion of individuals over 70 years old.

\author{
Corresponding author: \\ Amirhossein Sahebkar \\ PharmD, PhD \\ Mashhad University \\ of Medical Sciences \\ P.O. Box: $91779-48564$ \\ Mashhad, Iran \\ Phone: +985138002288 \\ Fax: +985138002287 \\ E-mail: \\ sahebkara@mums.ac.ir, \\ amir_saheb2000@yahoo.com
}




\section{Conflict of interest}

Maciej Banach has served on the speaker's bureau and as an advisory board member for Amgen, Sanofi, Aventis and Lilly. Dimitri P. Mikhailidis has given talks and attended conferences sponsored by MSD, Libytec and AstraZeneca.

\section{References}

1. The HPS3/TIMI55-REVEAL Collaborative Group. Effects of anacetrapib in patients with atherosclerotic vascular disease. New Engl J Med 2017; 377: 1217-27.

2. Madsen CM, Varbo A, Nordestgaard BG. Extreme high high-density lipoprotein cholesterol is paradoxically associated with high mortality in men and women: two prospective cohort studies. Eur Heart J 2017; 38: 2478-86.

3. Penson P, Long DL, Howard G, et al. Associations between cardiovascular disease, cancer and very low HDL cholesterol in the reasons for geographical and racial differences in stroke (REGARDS) study. Eur Heart J 2017; 38 (Suppl 1): ehx504.3104.

4. Otvos JD, Mora S, Shalaurova I, et al. Clinical implications of discordance between low-density lipoprotein cholesterol and particle number. J Clin Lipidol 2011; 5: 105-13.

5. Ference BA, Kastelein JJP, Ginsberg HN, et al. Association of genetic variants related to CETP inhibitors and statins with lipoprotein levels and cardiovascular risk. JAMA 2017; 18: 947-56.

6. Cholesterol Treatment Trialists' (CTT) Collaboration. Efficacy and safety of more intensive lowering of LDL cholesterol: a meta-analysis of data from 170000 participants in 26 randomised trials. Lancet 2010; 376: 1670-81.

7. Sabatine MS, Giugliano RP, Keech AC, et al. Evolocum$a b$ and clinical outcomes in patients with cardiovascular disease. N Engl J Med 2017; 376: 1713-22.

8. Banach M, Stulc T, Dent R, Toth PP. Statin non-adherence and residual cardiovascular risk: there is need for substantial improvement. Int J Cardiol 2016; 225: 184-96.

9. Banach M, Rizzo M, Toth PP, et al. Statin intolerance an attempt at a unified definition. Position paper from an International Lipid Expert Panel. Arch Med Sci 2015; 11: 1-23.

10. Rosenson RS, Baker S, Banach M, et al. Optimizing cholesterol treatment in patients with muscle complaints. J Am Coll Cardiol 2017; 70: 1290-301. 